\title{
NOTAS SOBRE LA SUPERVIVENCIA DE LA CASA DE BORDA
}

Por Clementina Diaz y de Ovindo

En la esquina de las calles, Avenida Francisco I. Madero y Bolívar, antiguamente llamadas de San Francisco y Coliseo Nuevo, se levanta la construcción del siglo xvir, conocida como Casa de Borda, por haber sido mandada edificar por el magnate mínero don José de la Borda, y terminada probablemente en 1775 .

De esta casa escribió Manuel Toussaint:

Casas de don José de la Borda (Madero, Bolivar, 16 de septiembre y Motolinía). El gran caballero español que nos ha donado tanto su fortuna, en la iglesia de Taxco, como su espiritu en estas caridades, tuvo la idea de construir una casa que ocuparía toda una manzana en la ciudad, como la propia de Cortés mucho antes. Debería estar limitada por las cuatro calles arriba mencionadas, y un largo y continuo balcón le permitira caminar alrededor de su propiedad. Este propósito nunca se realizó, pero varias casas en la actual Avenida Madero y la calle de Bolivar están unidas por un balcón como el antes mencionado.

La "Casa de Borda" como muchas construcciones coloniales, ha sufrido en el curso del tiempo transformaciones que alteraron de manera fundamental su primitiva fábrica. Hoy día sólo queda la fachada, a la que se agregaron dos pisos neocoloniales por el lado de Madero que destruyen la continuidad de lo que fue un ejemplar de casa palaciega metropolitana, en estilo barroco sobrio. La mansión es típica de la arquitectura civil del siglo xvm en la Ciudad de México, por la presencia del entresuelo, la prolongación de las jambas que enmarcan las cornisas; la combinación del tezontle para los muros y la chiluca para los elementos ornamentales y, sobre todo, la portada de acceso que presta al edificio grandeza y dignidad. La portada principal, que ha desaparecido, como correspondía al tono del xvill que daba tanta importancia a la composición de este elemento, debió ser de una sorprendente magnificencia.

Como testimonio de la ornamentación de las esquinas muy del gusto también del xvill, la Casa de Borda remata en un nicho que alberga una virgen guadalupana.

1 Colonial art in Mexico. University of Texas, Austin and London, 1967, p. 199. 
Las grandes fachadas de la Casa de Borda, ostentan aún parte de los hierros forjados originales que, según documentos que existen, fueron de primera calidad. Esos hierros forjados del gran balcón corrido del último piso otorgan al edificio un sentido de unidad monumental, el hermoso balcón parece indicar el deseo de don José de la Borda por pasear alrededor de su propiedad, como apunta Toussaint $\mathrm{y}$, también, el gusto de los propietarios por salir a contemplar la belleza del homogéneo paisaje urbano que presentaba la ciudad de México en el siglo xvir, así como solazarse con la animación que tenían las concurridas calles de San Francisco llamadas de Plateros, por ser las más opulentas en el comercio del rico metal.

La arquitectura interior de esta casa no puede juzgarse, ya que ha sido totalmente alterada, con esa incomprensión y menosprecio que tanto las autoridades oficiales como las eclesiásticas y civiles tienen por la obra de arte. La Casa de Borda, en 1908, estuvo en un tris de desaparecer, pues en su lugar se pretendía construir un hotel de lujo que, por fortuna, quedó en proyecto.

Creo que, para la historia del edificio, no está por demás conocer tanto el proyecto como lo que se afirmaba de esta casa palaciega en 1908, y por qué se consideraba que no era necesaria su conservación.

En la revista $E l$ arte y la ciencia de abril de 1902, se publicó el artículo del ingeniero civil y arquitecto Marian Téllez Pizarro, M.S.A., "Los progresos de la ciudad de Méjico en el último periodo de los ochenta y siete años", artículo en el que se señala el aumento de la propiedad urbana.

Para dar una idea breve -dice Téllez Pizarro- sobre los progresos de la ciudad de México desde 1814 hasta la actualidad, estimados por el aumento de valor de la propiedad urbana, que se relaciona directamente con el alza de precio de los terrenos que ocupan las fincas, presento una serie de lugares con los valores del metro cuadrado por cada uno en los años de 1814, 1872 y 1901 respectivamente.

\begin{tabular}{|c|c|c|c|c|}
\hline A.O.4. Call & de $\mathbf{P}$ & 16.02 & 40.50 & 150.00 \\
\hline " & idem & 14.24 & 38.00 & 135.00 \\
\hline " & 3a. de San Francisco & 12.46 & $\mathbf{3 4 . 0 0}$ & 110.00 \\
\hline " & 2a. idem & 10.60 & 26.00 & 90.00 \\
\hline$"$ & 1a. idem & $\mathbf{5 . 3 4}$ & 18.00 & 70.00 \\
\hline
\end{tabular}


Estos datos los he tomado de la tarifa de 1814 autorizada por el Ayuntamiento de la ciudad, revalidada en 1830 y modificada en 1848 por la misma corporación, autorizando un aumento en los precios hasta de un 50 por ciento a juicio del perito; de la tarifa de 1872 aprobada por la asociación de ingenieros y arquitectos de la ciudad de México, y de la que he calculado para 1901. Así se relacionan tres épocas que comprenden un período de ochenta y siete años.

La magnifica situación de la Casa de Borda hizo pensar seguramente a su propietario en la conveniencia de mayores rendimientos económicos pues, como se advierte en los datos de Téllez Pizarro; la propiedad urbana, que en esos años habian aumentado ostensiblemente, hacía que ya no fuera negocio el alquiler para comercios y oficinas.

En la Casa de Borda se encontraban el "Principal Gabinete Dental de México", del doctor norteamericano Marcos Fagg; la agencia de máquinas de coser "White" y los baños del "Harem" por la parte que daba a la calle del Coliseo. En la Casa Borda también estuvo el salón cinematográfico que se inauguró a mediados de agosto de 1906, según El Imparcial de 19 de agosto de ese año.

El local es bien amplio, fue decorado elegantemente y en él se arregló un foro, en el que durante los intermedios de las exhibiciones para darles mayores atractivos un grupo de artistas cantará romanzas de los mejores repertorios francés, italiano y español.

Tiene una variada y numerosa selección de vistas cinematográficas.

Las tandas comenzarán desde las cuatro de la tarde y durarán hasta las once de la noche.

Este salón cinematográfico a partir de noviembre de 1906 se llamó Salón Rojo y fue durante muchos años, hasta después de la Revolución, el cine más concurrido por las mejores familias de la capital, las que encontraban diversión, no sólo en las "vistas", sino también en los espejos cóncavos y convexos que alli había, y en subir por la entonces novedosa escalera eléctrica. Para los caballeros constituían un atractivo los aparatos con vistas estereoscópicas un "tanto atrevidas".

EI periódico El Imparcial, el 15 de marzo de 1908, consignaba una importante noticia con el título: "Un gran hotel en México". La capital -comentaba El Imparcial-tendria muy pronto un hotel con todas las exigencias modernas, que se llamaría muy francesamente "Hotel del

2 México, vol. 1, núm. 1, pp. $116-117$. 
Eliseo", construido y amueblado a todo lujo ya que se destinaba la cantidad de tres millones de pesos a este propósito.

Se ha alquilado para ejecutar la construcción el terreno que se halla en la esquina de San Francisco y Coliseo [Francisco I. Madero y Bolivar] por medio de un contrato que durará en vigor treinta años, con opción para la compra del propio terreno. Los signatarios de este contrato son los señores P. Bony y A. Gamard, actuales propietarios del Hotel Palacio. ${ }^{3}$

Los planos, afirmaba el periódico, habían sido aprobados y el contrato para la construcción del "Hotel del Eliseo", también estaba listo "entre Dubois, Morcon y Godan de esta ciudad".

El edificio se proyectaba de acero y concreto, de estilo moderno, con seis pisos y cuatrocientos departamentos. La demolición de los edificios darfa principio en treinta días a más tardar.

La entrada del "Hotel del Eliseo" sería por la esquina de las calles de San Francisco y Coliseo Nuevo y, además, tendría entradas en cada una de las calles laterales y dos escaleras cerca de estas entradas. Se instalarian dos elevadores para pasajeros y uno de carga.

En el primer piso - seguia informando El Imparcial- habrä un gran salón de baile,sala para recepción, sala privada para señoras, una cantina, salón para fumar y una peluquería. El resto de este piso será ocupado por un patio abierto y locales para oficinas. Los pisos superiores, serán los destinados para cuartos, recámaras y departamentos privados. En cada piso habrá, también, una sala privada para señoras. Los departamentos tendrán: teléfono propio, luz eléctrica y llaves de agua, caliente y fria; además habrá un departamento de baño anexo a cada cuarto.

Todos los departamentos darán a la calle o a patios abiertos, y se tendrá especial cuidado de que tengan bastante aire y luz de sol. Los muebles y tapices serán estilo Luis XVI, y se traerán expresamente de Paris.

En los sótanos habrá bodegas, en los que se guardarán los mejores vinos del mundo. Habrá también un planta eléctrica completa y refrigeradores para los alimentos. Se tendrán lavanderías, una estación de telégrafos y todas las comodidades que se tienen en los hoteles modernos de Europa y los Estados Unidos. Una de las innovaciones, desconocidas hasta ahora en México, será la calefacción del edificio por medio del vapor.

El nuevo hotel estará bajo la vigilancia personal del señor Gamard, que ha obtenido un éxito brillante en la administración del Hotel Palacio, éste permanecerá como un anexo del Palacio Eliseo.

s El Hotel Palacio estaba en la calle del Puente del Espiritu Santo, [Isabel la Católica] esquina con 16 de septiembre. 
Como todavía los automóviles eran escasos no se tomaron en cuenta las cocheras o garages, el estacionamiento que ahora se exige en los edificios modernos. Unos años después, el automóvil complicaría la vida de la ciudad, y contribuiría a la destrucción de ésta por la ampliación de calles y avenidas para la fluidez del tránsito, sacrificando monumentos de primer orden.

EI "Hotel del Eliseo", remedo de los de Europa, amueblado a todo lujo, seguramente hubiera sido más tarde, con la Revolución, destinado a otros menesteres; y sus dorados muebles, sus tapices, reducidos a cenizas. Sus salones si acaso, convertidos en oficinas, cabarets o salas té para disfrute de turistas; o mancillado también, como lo ha sido la Casa de Borda.

En 1908, el descontento en contra del régimen de Porfirio Díaz había empezado a manifestarse, aunque no se admitia, en manera alguna, su desplome. Sin hacer caso de esa inquietud, se continuaban con gran entusiasmo las obras para la celebración del primer centenario de la independencia de México, obras en las que se buscaban "soluciones plásticas irreprochables", que dieran a la ciudad de México el carácter de una capital europea. México seguía empeñado en el viejo ideal de ser como Europa.

La fotografia publicada por El Imparcial del 15 de marzo del proyecto para el "Palacio del Eliseo", es clara muestra de ese afán europeizante.

El "Palacio del Eliseo" iba a ser un ejemplo de arquitectura eclécticá; en el proyecto se advierten reminiscencias del neoclásico, combina. das al gusto francés que imperaba en esos años y que se identificaba estilisticamente con los nuevos edificios que se estaban construyendo para el centenario de la Independencia. Hay que decir que el "Palacio del Eliseo" estaba proyectado con bastante acierto y dignidad para las funciones a que estaba destinado.

E1 17 de marzo El Imparcial en su apartado "Hace veintiocho años valía doscientos mil pesos. Alli estuvo el primer sitio de coches públicos. Una ojeada al aumento de la propiedad urbana", proporciona más datos sobre la Casa de Borda. Según El Imparcial, un comerciante que durante diecinueve años habia sido inquilino del edificio; aseguraba que en el año de 1880 se le habian ofrecido doscientos mil pesos a su dueña, la señora esposa de don Felipe Robles, negándose ésta a venderlo, pues no tenía en qué emplear tanto dinero. 
"Y esa casa, que en 1880 valía doscientos mil pesos, se vendió últimamente -hace unos cuantos meses -en más de un millón."

El comprador y propietario de la Casa Borda fue Gabriel Fernández Somellera," quien la adquirió mediante la entrega de una hacienda y determinada cantidad de dinero.

El Imparcial sostenía que el edificio de la esquina de San Francisco y el Coliseo sólo tenia un relativo interés histórico.

No es casa de Historia.- Hay en México vetustos y casi ruinosos edificios que tienen una interesante historia, pues ya han sido mansión de algún notable personaje, ya se han cometido en ellos crimenes espeluznantes y han ocupado la atención pública por tal o cual suceso de importancia que en su interior se haya desarrollado.

Pero la casa que nos ocupa tiene relativa importancia histórica si es que alguna pueda tener el hecho de que fue en el patio de esa finca donde quedó establecido, por primera vez en México, un sitio de coches de alquiler, que cobraban cincuenta centavos por hora.

Después estuvo alli, por muchos años, el Casino Español, y más tarde todo el edificio se dividió en pequeños compartimientos que desde entonces acá se alquilan para despachos.

Como puede verse, el valor artístico del edificio pasó inadvertido para el periodista, autor del reportaje. La indiferencia ante Ia destrucción de esta casa colonial es evidente.

Unos años antes, el 30 de marzo de 1906, El Imparcial en su apartado "Los edificios de la época colonial" denunció la destrucción de las casonas coloniales, pero esta vez no protestó, como tampoco lo hicieron otros diarios.

La salvación de la Casa Borda al parecer que se debió a que los inquilinos de los comercios, situados en la planta baja del edificio, tenian contratos para cuyos vencimientos deblan transcurrir varios años $y$, aunque se les hicieron muy buenos ofrecimientos para anular los contratos

- En 1908, Fernández Somellerla compró a Feliciano Cobián en $\$ 860000.00$ la mansión conocida como "Palacio de Cobian" (esquina de Bucareli y General Prim, ahora Secretaria de Gobernación), obra del arquitecto Emilio Dondé, Dos affos despues, el 23 de junio de 1910, Fernández Somellera vende el palacio al Gobierno Federal en $\$ 990000.00$. Seguramente, Fernández Somellera que no fue hombre de negocion, debia demasiadas contribuciones, y eae debe haber sido el motivo por el que se le redujo el precio. En 1913 durante la época de Victoriano Huerta, Fernándea Somellera fue presidente del Partido Católico. Ia enorme fortura de este representante de los ricos porfirianos, hecha con las haciendas algodoneras acabo esfumándone. 
y salir de la casa, seguramente no aceptaron; después vino la Revolución y el proyecto, por suerte, no pudo realizarse, pues se hubiera perdido un importante monumento de arquitectura civil como lo es por su historia y belleza la Casa Borda.

En mayo de 1972 la "Casa de Borda" fue declarada por ministerio de ley: monumento. En ese mismo año el Departamento del Distrito Federal inició las obras para rehabilitar el centro de la ciudad: restauración y limpieza de fachadas, cambio de pavimento, instalación de alumbrado. La primera avenida que se rehabilitó fue la de Francisco I. Madero, así la Casa Borda tiene un mejor marco para el lucimiento de sus fachadas. 
DOI: http://dx.doi.org/10.22201/iie.18703062e.1974.43.995 


\section{[osscientos Mil Pesos}

Alli Lśtuvo el Friner Silio de Coches Públics. Una

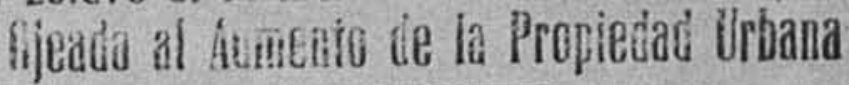

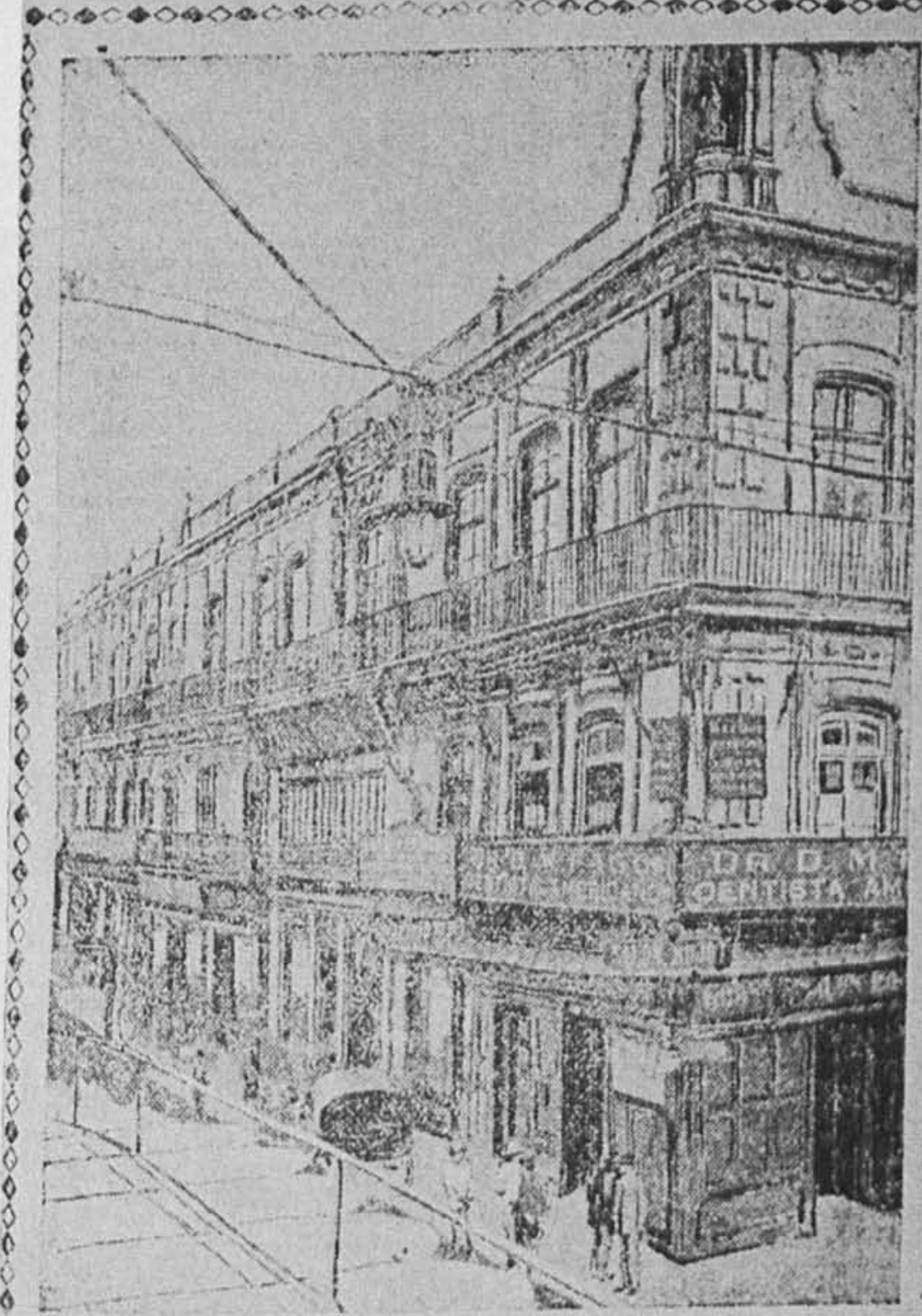

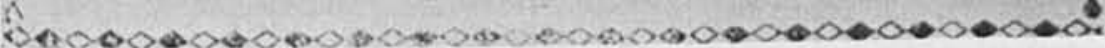

1. Casa de Borda en 1908. El Imparcial, México, 17 de marzo de 1908 


\section{JEN GRADN HOIEL EN MEXICO}

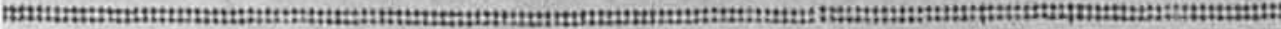

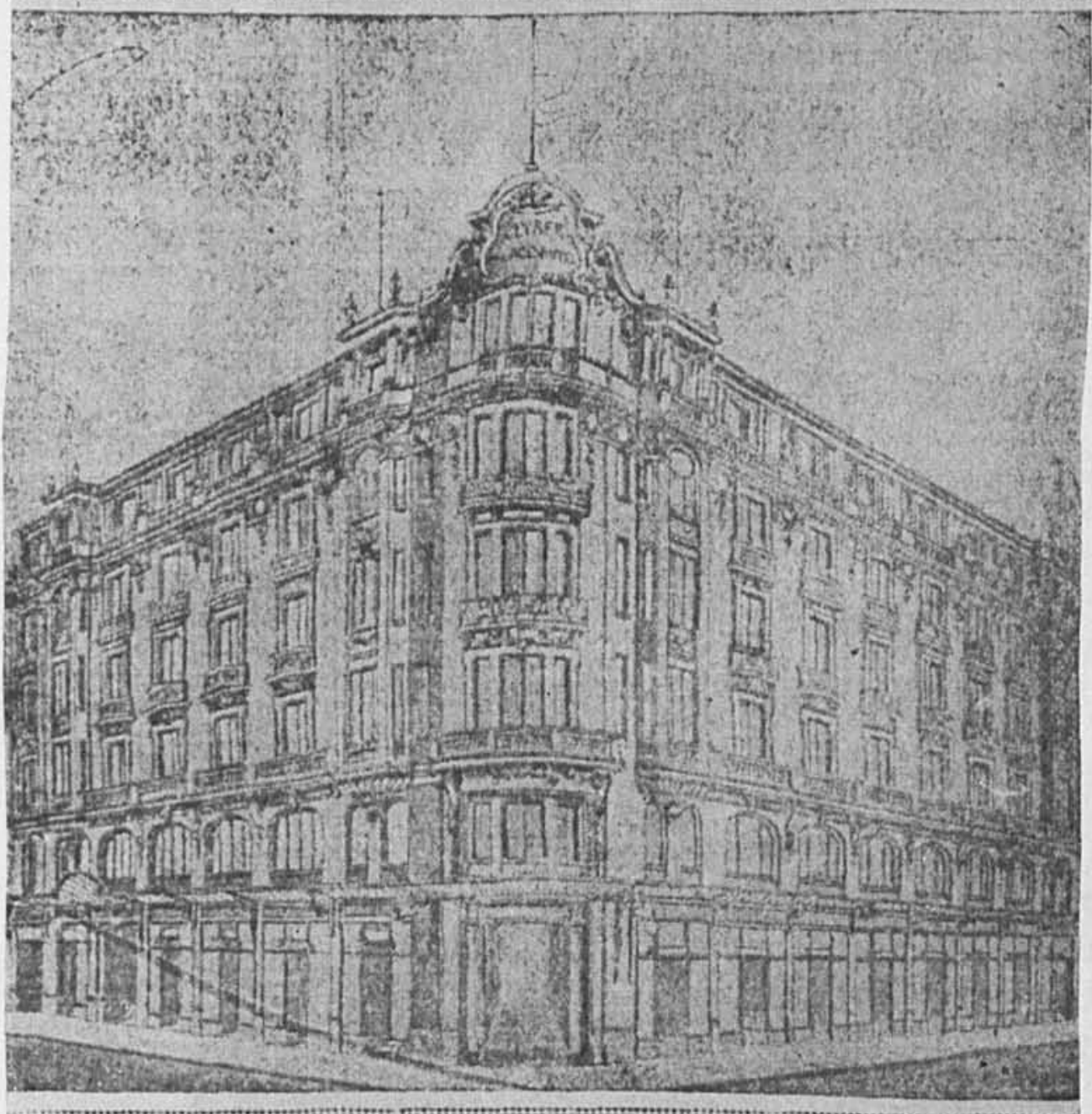

2. Proyecto para el Hotel del Eliseo. El Imparcial, México, 15 de marzo de 1908 
DOI: http://dx.doi.org/10.22201/iie.18703062e.1974.43.995

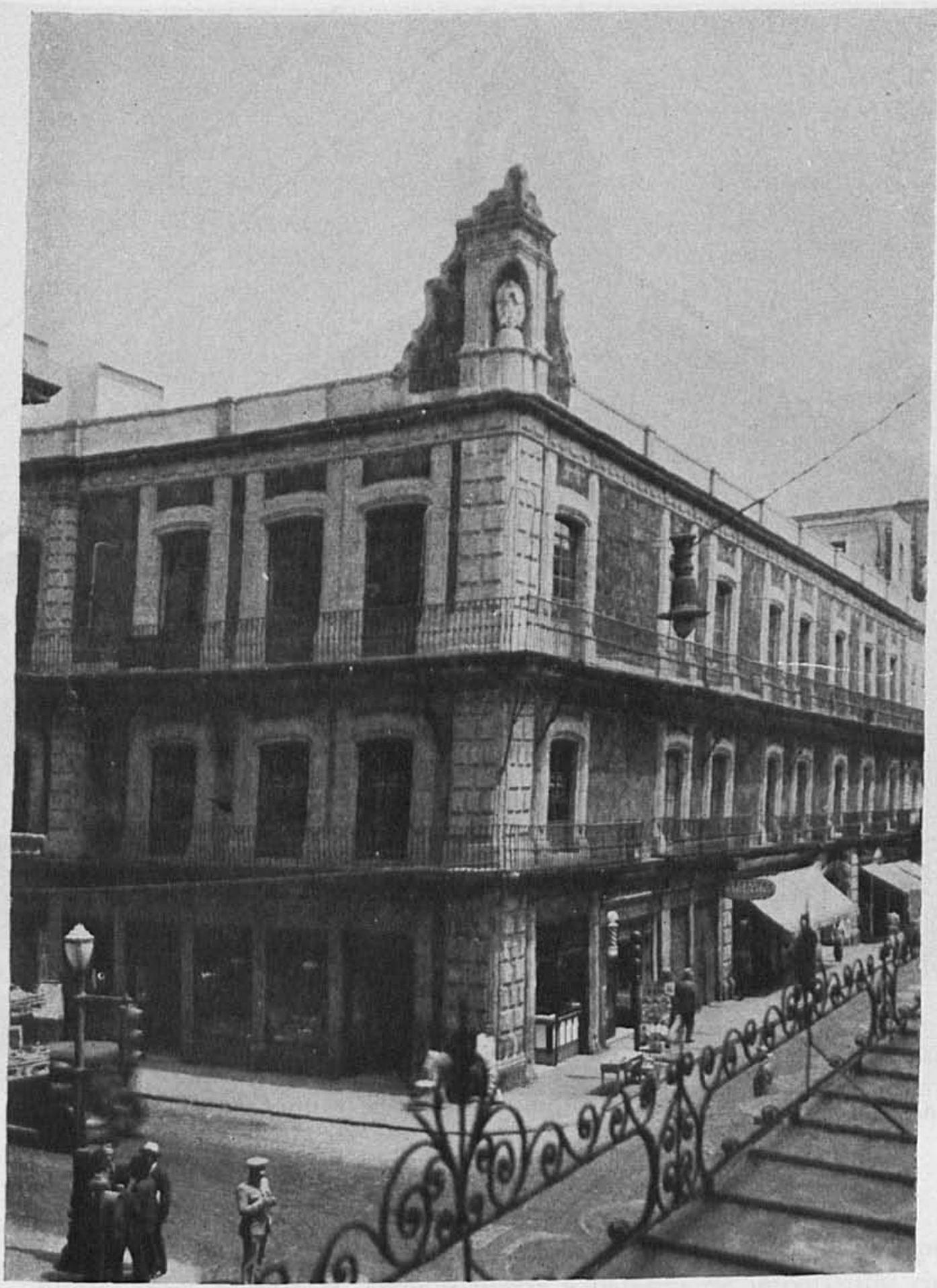

3. Casa de Borda, con los dos niveles añadidos posteriormente sobre Ia fachada norte 


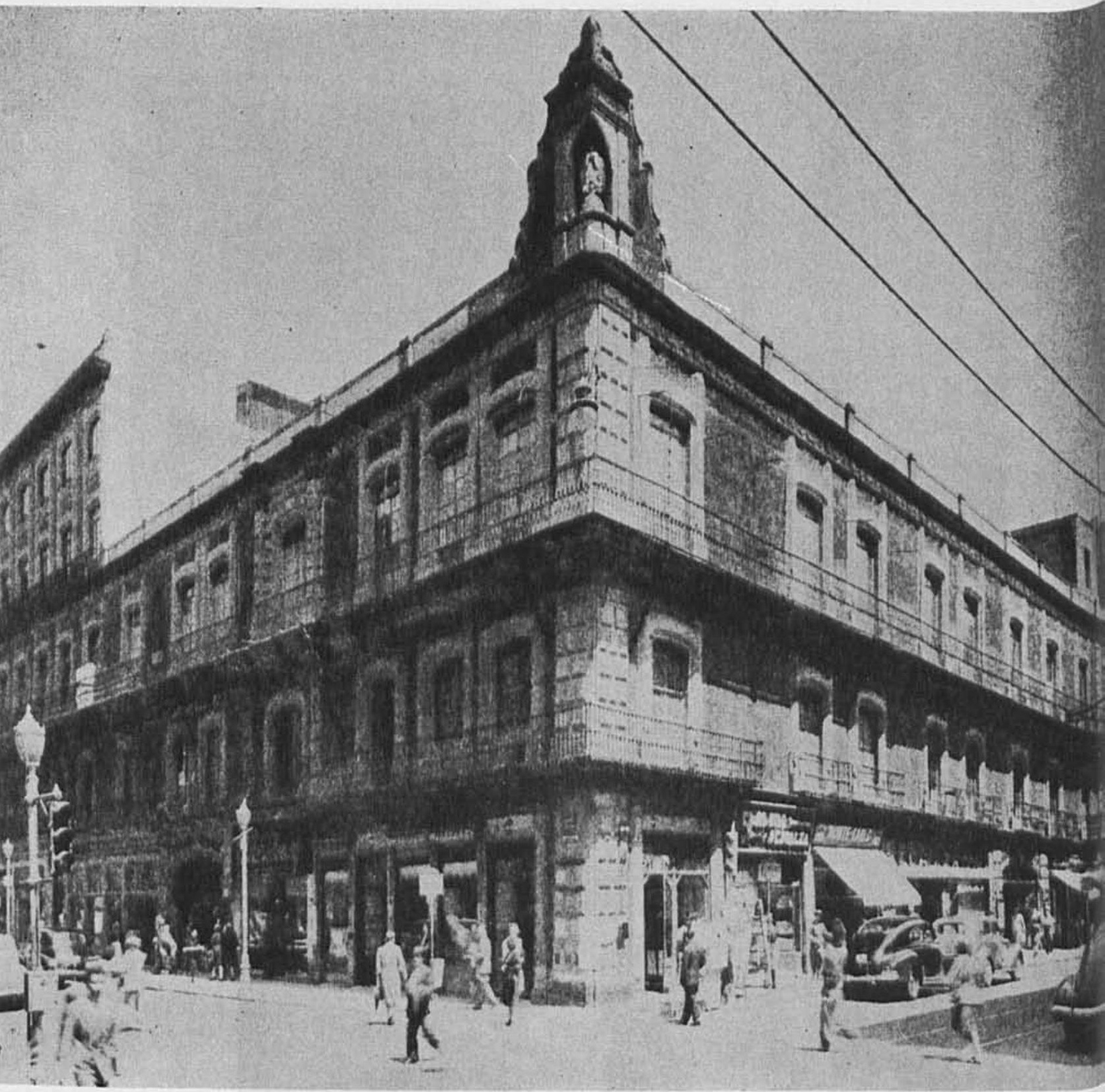

4. Casa de Borda. Iistado actual 